\title{
Reconfigurable parasitic antennas for compact mobile terminals in multiuser wireless systems
}

\author{
Vlasis I Barousis ${ }^{1}$, Athanasios G Kanatas ${ }^{1 *}$, Antonis Kalis ${ }^{2}$ and Julien Perruisseau-Carrier ${ }^{3}$
}

\begin{abstract}
This article considers the exploitation of parasitic antenna arrays in multi-user (MU) wireless communication systems by using their adaptive beamforming capabilities in order to improve the average system throughput. The use of parasitic arrays and especially the electrically steerable passive array radiator (ESPAR) antennas enables the design of terminals with a single RF front-end and reduced antenna dimensions, i.e., lightweight and compact mobile terminals. Although the beamforming capabilities of active element arrays at the receiver have been well investigated in the past, this article highlights the potentials of pattern reconfigurable parasitic arrays based on the beamspace representation of the ESPAR antenna. The advantages of using ESPAR at the receiving terminal are examined both in opportunistic beamforming and in MIMO broadcast channel MU systems, optimizing correspondingly the SNR or the SINR of the forward link.
\end{abstract}

\section{Introduction}

The use of multi-element antenna arrays has proven to be an effective means of turning multipath propagation to an advantage in wireless communication systems, by exploiting the diverse propagation characteristics of multipath components to increase the robustness of communication through diversity techniques, or the capacity of wireless links through spatial multiplexing of multiple symbol-streams. Recently, Knopp and Humblet [1] have used the same properties of multi-element array systems in multi-user (MU) environments, focusing on the reverse channel of cellular communication systems, where a large number of users, each equipped with a single antenna, access a single base station (BS) through a time-varying wireless channel. In their study, they prove that the average throughput of the system is maximized when the BS grants access to the user with the highest channel gain. The same results apply to the forward link [2]. The main idea in opportunistic beamforming scenarios is the use of a different radiation pattern at the BS at each TDMA time slot, in order to induce a time-varying environment, even in the case of slow fading conditions. This idea could be implemented with the use of a multiple antenna array at the BS,

\footnotetext{
* Correspondence: kanatas@unipi.gr

'Department of Digital Systems, University of Piraeus, 80 Karaoli \& Dimitriou St., 18534, Piraeus, Greece

Full list of author information is available at the end of the article
}

which would produce a random radiation pattern on each TDMA time slot [3]. If the BS had full Channel State Information (CSI) for all users at all times, then it could optimize the radiation pattern in order to maximize the signal to noise ratio (SNR) at each user. However, since full CSI knowledge would require excessive use of the channel resources for exchange of CSI information through feedback from the users to the BS, in practice the BS only requests for SNR level information from the users, which is inadequate for optimal beamforming. With opportunistic beamforming, due to the large number of users within a single cell, a random radiation pattern created on each user time slot would be close to the optimum radiation pattern for at least one user, with high probability. That user with the highest SNR would therefore be granted access on that time slot.

The use of pattern-reconfigurable antennas for improved capacity is not a new idea and actual implementations have been presented in [4-6]. The idea in these studies consists in enabling the dynamic reconfiguration of the antenna radiation patterns to provide some level of dynamic control over the channel itself. The antenna property, namely its instantaneous 'state', is thus an additional degree of freedom that can be optimized at each time slot by the algorithm implementing the smart antenna capability. As a result, this concept applies to both beamforming and MIMO schemes. It is

\section{Springer}


also worth mentioning that here 'pattern reconfiguration' refers to the control of both polarization and spatial power spectrum of the radiated field since both these parameters affect channel property. In [5], Du and Gong present an operational antenna for $2 \times 2$ MIMO but do not assess its impact on the capacity. In [6], the mutual coupling between the two antenna elements for $2 \times 2$ MIMO is dynamically controlled, which in turn affects their radiation patterns (indeed, it can be shown that the coupling between antennas is directly related to their radiation patterns). For SNR of $10 \mathrm{~dB}$ and $20 \mathrm{~dB}$, $10 \%$ and $8 \%$ capacity improvements are obtained with respect to a non reconfigurable system. In [4], the effect of both antenna diversity and gain in $2 \times 2$ MIMO are evaluated at SNR of $10 \mathrm{~dB}, 20 \mathrm{~dB}$, and $30 \mathrm{~dB}$, leading to capacity improvements of $70 \%, 40 \%$, and $26 \%$, respectively. However, the capacity gains achieved strongly depend on the test scenario. The approach in these studies essentially consists of designing some reconfigurable antennas with a certain level of pattern diversity, and subsequently evaluate the impact of this capability on the capacity. As explained in detail in the remainder of this article, here a more advanced strategy is proposed by exploiting the particular nature of parasitic array antennas, and in particular the decomposition of their instantaneous reconfigurable patterns onto a basis of orthogonal functions.

In [7-9], it was clearly shown that parasitic array antennas preserve the capability to also perform MIMO transmission. Therefore, the design of single RF frontend MIMO terminals is feasible, [10], and efficiently addresses the long experienced limitations imposed by the physical size of the terminals. The existence of a single active port motivates the representation of the MIMO functionality at the beamspace domain, where diverse symbols are mapped to different basis patterns. Indeed, the degrees of freedom (DoFs) of the electrically steerable passive array radiator (ESPAR) antennas have been explored by providing the expansion of the far field pattern in a complete set of orthonormal basis functions, or basis patterns. The operation was initially described in [11] and then a generalized and analytic methodology was presented in $[12,13]$. This alternative analysis takes advantage of the beamforming capabilities provided by the parasitic elements that are connected to tunable loads, and determines the DoFs at the beamspace domain. Thus, single port antennas with beamforming capabilities can be used to emulate MIMO transmission. The significantly reduced antenna dimensions, as well as the single RF chain required to support diversity and multiplexing capabilities, are the enabling characteristics of parasitic antennas for lightweight and compact mobile terminals. The use of electronically steerable parasitic antennas is not the only way to get compact, lightweight and low cost MIMO transceivers. Recently, a novel MIMO scheme based on analog combining has been explored in depth [14-18]. This MIMO architecture solves the implementation complexity by shifting spatial signal processing from the baseband to the radio-frequency (RF) front-end and is known as RFMIMO. The basic idea of the RF-MIMO transceiver is to perform adaptive signal combining in the RF domain. After combining, a single stream of data must be acquired and processed, thereby reducing cost and power consumption as compared to the conventional MIMO scheme with multiple active streams. An experimental evaluation of the RF-MIMO concept can be found in [19]. Although this scheme has been shown to provide full diversity and array gain, its multiplexing gain is limited to one, as a result of processing a single data stream. In contrast, ESPAR based MIMO provide multiplexing gain thanks to the novel aerial modulation technique. However, RF-MIMO architecture has been shown to support OFDM schemes, while ESPAR based MIMO support to the moment single carrier transmission. Other similarities and differences between RFMIMO and ESPAR based MIMO concern the beamforming process and are reviewed in [20].

The major contribution of this study is the use of recent developments in reconfigurable parasitic arrays and in the beamspace representation of their patterns, in order to optimize the performance of the forward link in opportunistic beamforming and MIMO broadcast channel MU systems. The presentation of our findings is organized in the following sections. In Section 2, we present a review of reconfigurable parasitic antenna technologies with emphasis on their feasibility and adaptive capabilities, which enable the analysis of this article. Section 3 presents the advantages of using parasitic arrays on mobile terminals in opportunistic beamforming multiuser scenarios, while Section 4 presents the respective gains achieved in MIMO broadcast channel MU scenarios. Section 5 concludes the results of this research activity. One paragraph describing paper contents and contribution. (Actually in opportunistic beamforming MU systems and in MU-MIMO broadcast channels).

\section{Reconfigurable parasitic antennas for lightweight terminals}

Multiple antenna arrays have been for long considered for increasing the wireless link performance in applications where the size and cost of their implementation is not restrictive. Indeed, multi-element arrays have been widely used in BSs, but their implementation in mobile terminals is restricted by the available real estate for the antennas and the need for separate RF chain for each antenna element (except if the array is used to achieve 
SISO beamforming only). Although the size issue can be quite efficiently tackled by the use of 'orthogonal resonant modes', see e.g., [21], the burden of the multiple RF chains remains.

Recently, a novel parasitic array architecture has been developed [21-23], which can significantly decrease the size and cost of arrays, thus making their integration in handheld terminals feasible. These arrays consist of only a single active element and a number of parasitic elements placed in close proximity. Due to strong mutual couplings, the feeding of the active element is responsible for the currents induced to all parasitics. The dynamic control of the parasitic array radiation patterns is performed directly in baseband, through the dynamic control of passive reactive loads connected directly to all parasitics and thus altering mutual coupling and antenna radiation characteristics [24]. Importantly for practical designs, the complete description of the parasitic array performance (return loss, efficiencies, patterns, etc.,) in all possible dynamic states, can be computed based on a single electromagnetic full-wave simulation followed by simple post-processing [23]. It is of course of prime importance to precisely implement the reactive loads, as detailed in [10]. So far, their control has been achieved using varactors or $\mathrm{p}-\mathrm{i}-\mathrm{n}$ diodes. However, as in other applications the use of MicroElectroMechanical Systems (MEMS) would result in better performance in terms of insertion loss, linearity, while having virtually zero DC power consumption. In this study, the simulation results presented in the following sections we have not assumed a specific implementation technique but we have restricted our interest to the values of the loads and the corresponding radiation characteristics of the antenna.

Traditionally parasitic array implementations focused on SISO beamforming, since the use of a single RF port constrained them from being used in MIMO systems. In this sense, they present a similar functionality as conventional arrays achieving beamforming through analog RF phase shifters. However, recently such parasitic array systems have been effectively used in MIMO systems simultaneously transmitting multiple bit streams over the air, through the decomposition of their instantaneous reconfigurable patterns onto a basis of orthogonal functions. As will be shown, the resulting radiation pattern is the linear combination of the baseband symbols and the basis patterns and can be viewed as creating multiple symbol streams at the beamspace domain. To emphasize its principle of operation, the resulting single RF MIMO system is known as beamspace MIMO (BSMIMO). It should be noted that this MIMO approach takes advantage of the coupling between the adjacent
ESPAR elements. Indeed, the strong coupling enables the beamforming capability, which in turn is required to emulate MIMO transmission over the air $[12,13]$. In fact this idea has already been quite extensively exploited on the transmitter side, from the initial concept presented in [9] and the detailed design of the actual reconfigurable parasitic antenna and experimental demonstration in [10]. These studies demonstrated the tremendous advantages of using ESPAR antennas at the transceiver, since it was shown both theoretically and experimentally that a single ESPAR with a particular feeding scheme allows to multiplex data while using a single antenna and RF chain $[9,10]$.

In this new contribution we evaluate the benefits of using the beamforming capabilities of parasitic array antennas at the receiver side, by exploiting the orthonormal expansion of the far field pattern of ESPAR antenna in a complete set of basis functions. The methodology is based on the well known Gram-Schmidt orthonormalization procedure, which provides a 3D orthogonal expansion of the beamspace domain of the antenna. As explained in detail in [12,13], the radiation pattern of an ESPAR antenna with one active and ( $M$ 1) parasitic elements is given by

$$
P(\theta, \varphi)=\mathbf{i}^{T} \mathbf{a}(\theta, \varphi)=\sum_{m=0}^{M-1} i_{m} a_{m}(\theta, \varphi)
$$

where $\mathbf{a}(\theta, \varphi)=\left[a_{0}(\theta, \varphi) \ldots a_{M-1}(\theta, \varphi)\right]^{T}$ is the steering vector of the ESPAR at a direction $(\theta, \phi)$, and $\mathbf{i}$ is the current vector given by $\mathrm{i}=v_{s}\left(\mathbf{Y}^{-1}+\mathbf{X}\right)^{-1} \mathbf{u}$. The admittance matrix $\mathbf{Y}$, is an $(M \times M)$ matrix obtained by using an antenna analysis software, and each entry $y_{i j}$ represents the mutual admittance between the $i$ th and $j$ th element. The load matrix $\mathbf{X}=\operatorname{diag}\left[\begin{array}{llll}50 & j x_{1} & \ldots & j x_{M-1}\end{array}\right]$, adjusts the radiation pattern, whereas $\mathbf{u}=[10 \ldots 0]^{T}$ is a $(M \times 1)$ column selection vector and $v_{S}$ is the complex feeding at the active element. To represent $P(\theta, \phi)$ at the beamspace domain, the functions $a_{m}(\theta, \phi), m=0$, ..., $M-1$ are expressed as a linear combination of orthonormal functions $\Phi_{n}(\theta, \phi)$. For this purpose, the process of Gram-Schmidt orthonormalization is used providing:

$$
P(\theta, \varphi)=\sum_{n=0}^{M-1} \mathbf{i}^{T} \mathbf{q}_{n} \Phi_{n}(\theta, \varphi)=\sum_{n=0}^{M-1} w_{n} \Phi_{n}(\theta, \varphi)
$$

where $\mathbf{q}_{n}=\left[q_{0 n} \ldots q_{(M-1) n}\right]^{T}$. contains the projections of all functions $a_{m}(\theta, \phi)$ onto $\Phi_{n}(\theta, \phi)$. From (2) the $n$th basis pattern is weighted by the symbol $w_{n}=\mathbf{i}^{T} \mathbf{q}_{n}$ and $\mathbf{w}=\left[\begin{array}{llll}w_{0} & w_{1} & \ldots & w_{M-1}\end{array}\right]^{\mathbf{T}}$ defines a coordinate vector at 
the beamspace domain which corresponds to a radiated pattern. For a circular ESPAR with 5 elements, the basis patterns that construct the beamspace domain are given by [13]

$$
\begin{aligned}
& \Phi_{0}(\theta, \varphi)=\frac{1}{k_{0}} \quad \Phi_{1}(\theta, \varphi)=\frac{1}{k_{1}} \sin (b \sin \theta \cos \varphi) \\
& \Phi_{2}(\theta, \varphi)=\frac{1}{k_{2}} \sin (b \sin \theta \sin \varphi) \Phi_{3}(\theta, \varphi)=\frac{1}{k_{3}}\left[\cos (b \sin \theta \cos \varphi)-\frac{q_{30}}{k_{0}}\right] \\
& \Phi_{4}(\theta, \varphi)=\frac{1}{k_{4}}\left[\cos (b \sin \theta \sin \varphi)-\frac{q_{40}}{k_{0}}-\frac{q_{43}}{k_{3}} \cos (b \sin \theta \cos \varphi)+\frac{q_{43} g_{30}}{k_{0} k_{3}}\right]
\end{aligned}
$$

where $b=2 \pi d$, and $d$ is the normalized to the wavelength distance of the parasitics from the active element. Moreover,

$k_{n}=\sqrt{\int_{0}^{2 \pi} \int_{0}^{\pi}\left|a_{n}(\theta, \varphi)-\sum_{s=0}^{n-1} q_{n s} \Phi_{s}(\theta, \varphi)\right|^{2} \sin \theta d \theta d \varphi}$, are the normalization coefficients ensuring basis patterns with unit power, and $q_{m n}$ are the projections given by

$$
\begin{aligned}
& q_{30}=\frac{\pi}{k_{0}} \int_{0}^{2 \pi} E_{1}(b \cos \varphi) d \varphi \quad q_{40}=\frac{\pi}{k_{0}} \int_{0}^{2 \pi} E_{1}(b \sin \varphi) d \varphi \\
& q_{43}=\frac{\pi}{k_{3}} \int_{0}^{2 \pi} E_{1}[2 b \cos (\pi / 4) \cos \varphi] d \varphi-\frac{q_{30} q_{40}}{k_{3}}
\end{aligned}
$$

and the function $E_{1}(z)$ denotes the Weber function of the first order defined as [25]

$$
E_{v}(z)=\frac{1}{\pi} \int_{0}^{\pi} \sin (v \theta-z \sin \theta) d \theta
$$

Examples of radiation patterns can be found in $[12,13]$.

The MIMO functionality is presented at the beamspace domain. At the transmitter, symbols are not driven to diverse active antenna elements as in conventional case, but they modulate the orthogonal radiation patterns of the basis. The presented decomposition implies that the number of DoFs, i.e., the beamspace dimensionality, is equal to the number of ESPAR elements. However in $[12,13]$ it was shown that the electromagnetic coupling between the ESPAR elements, which is heavily dependent on the antenna dimensions, strongly affects the subset of significant DoFs, $N_{\text {eff }} \leq M$, called effective DoFs (EDoFs).

\section{SNR optimization in opportunistic beamforming systems}

The idea of opportunistic beamforming has shown that in MU environments, fading is actually a desired property of the wireless channel. Opportunistic beamforming will therefore improve the performance of wireless channels having a strong line-of-sight (LoS) component (i.e., Rician channels), by transforming them into severely faded channels. In this section, we enhance this idea by introducing the use of multi-element arrays on the receiver side, in order to maximize the received signal's SNR. We consider two different cases of static channels: Rayleigh and Rician. In the former case, it has already been shown that opportunistic beamforming has no enhancing effects of the average system throughput. Therefore, for Rayleigh channels we only consider the optimal beamforming scenario on the receiver side. In the case of Rician channels we examine the enhancement of average network throughput when in addition to the opportunistic beamforming at the BS, switching is performed at the receiver among different radiation patterns having significant antenna gains.

\subsection{System model}

The channel matrix of a link between a BS with $M_{T}$ antenna elements and a handheld terminal equipped with a parasitic array providing $N_{e f f, u}$ DoFs is given by

$$
\mathbf{H}^{(u)}=\boldsymbol{\Phi}_{u}^{H} \mathbf{H}_{g}^{(u)} \boldsymbol{\Phi}_{T}
$$

where $\mathbf{H}_{g}^{(u)}$ is a diagonal matrix with the channel complex gains of $Q$ multipath components, $\boldsymbol{\Phi}_{u}$ is a $(Q \times$ $N_{\text {eff, u }}$ ) sized matrix, with the $i$ th column having the array response vector of the $i$ th basis radiation pattern towards the directions of the scatterers. Similarly, $\boldsymbol{\Phi}_{T}$ describes the array response vectors of the BS. At the beginning of each time frame, the BS executes an opportunistic beamforming algorithm for defining the random radiation pattern with weight vector $\mathbf{w}_{T}$, of dimensions $\left(M_{T} \times 1\right)$. The complex gain of the $u$ th user channel is equal to

$$
h^{(u)}=\mathbf{w}_{u}^{H} \boldsymbol{\Phi}_{u}^{H} \mathbf{H}_{z}^{(u)} \boldsymbol{\Phi}_{T} \mathbf{w}_{T}=\mathbf{w}_{u}^{H} \tilde{\mathbf{h}}^{(u)}=\sum_{i=1}^{N_{e f f, u}} w_{u, i} \tilde{h}_{i}^{(u)}
$$

where $\tilde{h}_{i}^{(u)}$ is the $i$ th element of the vector $\tilde{\mathbf{h}}^{(u)}=\boldsymbol{\Phi}_{u}^{H} \mathbf{H}_{g}^{(u)} \boldsymbol{\Phi}_{T} \mathbf{W}_{T}$ with dimensions $\left(N_{\text {eff }, u} \times 1\right)$ and $\mathbf{w}_{u}=\left[w_{u, 1}^{*} w_{u, 2}^{*} \ldots w_{u, N_{e f f, u}}^{*}\right]$ is a complex weight vector describing the receiving instantaneous/effective pattern as a function of the basis functions (See Section 2). The received signal may then be written as:

$$
\gamma^{(u)}=h^{(u)} s^{(u)}+n^{(u)}=\mathbf{w}_{u}^{H} \boldsymbol{\Phi}_{u}^{H} \mathbf{H}_{8}^{(u)} \boldsymbol{\Phi}_{T} \mathbf{w}_{T s^{(u)}}+n^{(u)}=\mathbf{w}_{u}^{H} \tilde{\mathbf{h}}^{(u)} s^{(u)}+n^{(u)}
$$

where $s^{(u)}$ and $n^{(u)}$ are the transmitted signal and the Gaussian noise for user $u$, respectively.

\subsection{Receiver beamforming in Rayleigh channels}

In order to define its optimal radiation pattern, each user needs to have full knowledge of the channel matrix 
$\mathbf{H}^{(u)}$. In conventional array systems with multiple active elements, this is achieved by the transmission of a single training sequence per transmit antenna or transmit radiation pattern. However, in the cases considered in this article, where switched parasitic arrays are used at the receiver side, for each radiation pattern used at the transmitter side, the user has to receive $N_{\text {eff, } u}$ training sequences, for estimating the complex response of every basis pattern of the receive antenna, thus forming the matrix $\mathbf{H}^{(u)}$. The problem of maximizing the SNR at the receiver corresponds to the problem of maximizing the received signal strength at each user, described as,

$$
g_{u}=\max _{\mathbf{w}_{u}}\left|\mathbf{w}_{u}^{H} \tilde{\mathbf{h}}^{(u)}\right|^{2}=\max _{\mathbf{w}_{u}}\left(\mathbf{w}_{u}^{H} \mathbf{D}_{u} \mathbf{w}_{u}\right)
$$

where $\mathbf{D}_{u}=\tilde{\mathbf{h}}^{(u)}\left(\tilde{\mathbf{h}}^{(u)}\right)^{H}$. The autocorrelation matrix $\mathbf{D}_{u}$ is of rank one, with only one non-zero eigenvalue $\xi$, the optimal weight vector of user $u$ is given by [26],

$$
\mathbf{w}_{u, o p t}=\sqrt{\frac{1}{\xi}} \tilde{\mathbf{h}}^{(u)}
$$

This equation is similar to the maximal ratio combining (MRC) technique in receive diversity applications $[27,28]$, using a single antenna at the transmitter and conventional arrays with multiple active elements at the receiver. Therefore, using parasitic arrays on handheld terminals can achieve comparable results to the use of multiple active elements, with the difference that in the former case the algorithm is performed on the beam-space domain, instead of the traditional antenna domain. In Figure 1, we show the effect of this technique on the average network throughput for parasitic arrays capable of producing 3 or 5 orthogonal basis patterns, compared to using conventional multi-

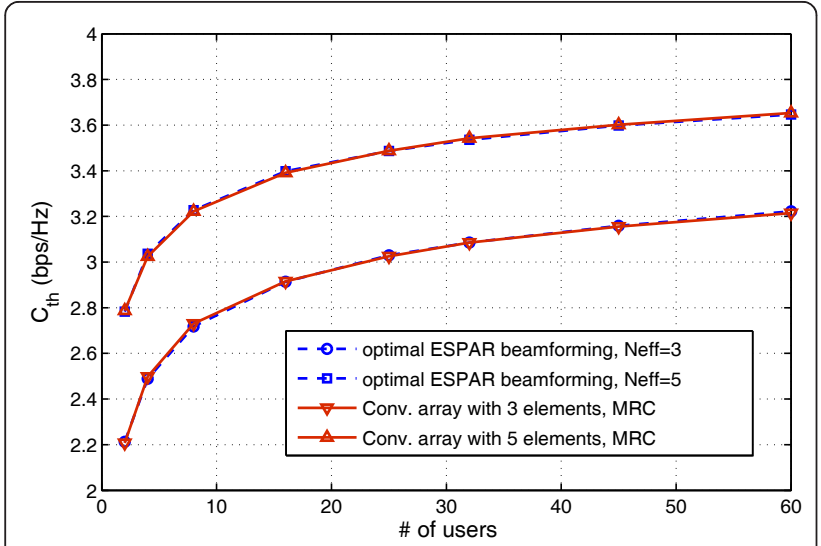

Figure 1 Optimal beamforming at the receiver with parasitic antennas. Effect of using parasitic antennas with optimal beamforming at handheld terminal receivers. element receive antennas and implementing MRC algorithms on mobile terminals. The average throughput has been computed by means of the following equation:

$$
C_{t h}=E\left[\log \left(1+\gamma\left(\max _{u=1 \ldots U}\left\{\left|h^{(u)}\right|^{2}\right\}\right)\right)\right]
$$

Equation 11 holds when on each time slot, and for time invariant channels within this slot, the user with the highest channel gain is selected. Therefore, using ESPAR antennas on mobile terminals would result in the same performance characteristics as in the case of having conventional multi-element arrays, while preserving the low-cost and small size characteristics of handheld devices. As shown in Figure 2, these lower complexity algorithms come at the cost of lower performance characteristics. It is also evident that random pattern selection at the receiver would have the same performance in Rayleigh channels, regardless of the number of effective DoFs, as expected by the analysis in $[2,3]$. Although the use of optimal beamforming algorithms with ESPAR antennas at the receiver can theoretically give performance gains equal to the use of traditional smart antennas, there is a key difference between the two systems that has to be considered. As mentioned above, in the case of ESPAR antennas, due to the fact that only one RF chain is used, in order for the receiver to acquire full channel knowledge, the training duration must be extended $N_{\text {eff, } u}$ times, so that ESPAR receivers have the time to switch among the $N_{\text {eff, }}$ ${ }_{u}$ different basis patterns. The extension of the training period has to be accounted for the analysis of the proposed solution, as described in the following. Assume that the downlink channel is time invariant (and we therefore need only a single training sequence) for $T_{\text {tot }}$

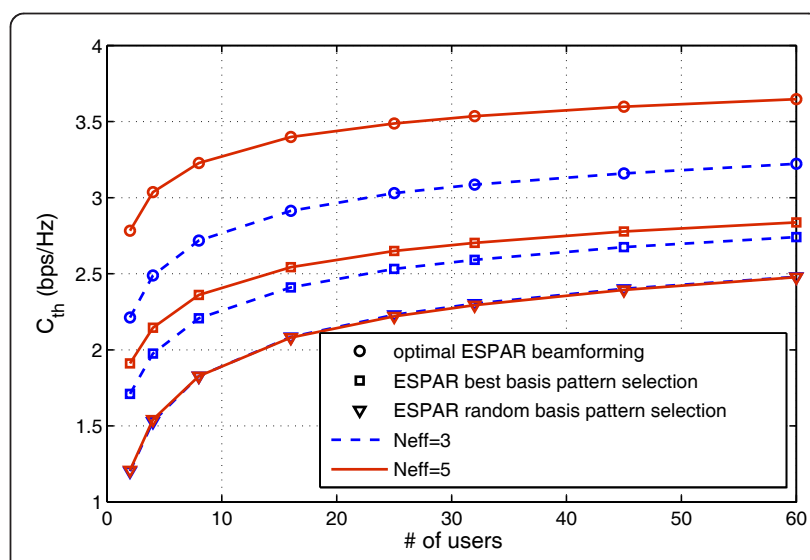

Figure 2 ESPAR antenna receiver algorithms. Comparison of different ESPAR antenna receiver algorithms. 
periods. Then the average channel goodput can be expressed as:

$$
C_{t h, a c t}=\left(1-\frac{T_{\text {train }}}{T_{\text {tot }}}\right) C_{t h}
$$

where $T_{\text {train }}$ is the number of required training periods per user. For a single BS radiation pattern, in conventional smart antenna systems we would have $T_{\text {train }}=1$, while ESPAR antenna terminals would need $T_{\text {train }}=N_{\text {eff, }}$, ${ }_{u}$, for acquiring the same channel information. Figure 3 shows that for relatively slow fading channels $\left(T_{t o t}>\right.$ $100)$, the difference between the two approaches is negligible, as expected. However, when the time variance of the channel increases, the effect of the increased training overhead is evident in the system performance.

\subsection{Receiver beamforming in Rician channels}

In order to evaluate the potential gains of using ESPAR antennas on mobile terminals in multiuser Rician channels we consider that both the BS and the mobile terminals perform opportunistic beamforming. This is performed by randomly selecting a pattern among those with the highest directivity that their antennas can produce. In this case, the channel between the BS and a mobile user can be expressed as,

$$
h_{u}=\underbrace{\sqrt{\frac{1}{1+K}} \mathbf{p}_{u, R}^{H} \mathbf{H}_{g}^{(u)} \mathbf{p}_{T}}_{\text {multipath fading }}+\underbrace{\sqrt{\frac{K}{1+K}} \mathbf{p}_{u, R}^{H}(i) \mathbf{p}_{T}(j) e^{j \phi_{u}}}_{\text {artificial fading }}
$$

where $\mathbf{p}_{T}=\boldsymbol{\Phi}_{T} \mathbf{w}_{\text {opt }}=\sum_{i=1}^{N_{\text {eff } T} T} w_{T, i, o p t} \boldsymbol{\varphi}_{T, i}$ is the vector of the azimuth samples of the transmit radiation pattern, while the column vectors $\phi_{T, i}$ of matrix $\boldsymbol{\Phi}_{T}=\left[\boldsymbol{\varphi}_{T, 0} \boldsymbol{\varphi}_{T, 1}, \ldots, \boldsymbol{\varphi}_{T, N_{e f f}-1}\right]$ contain the samples of

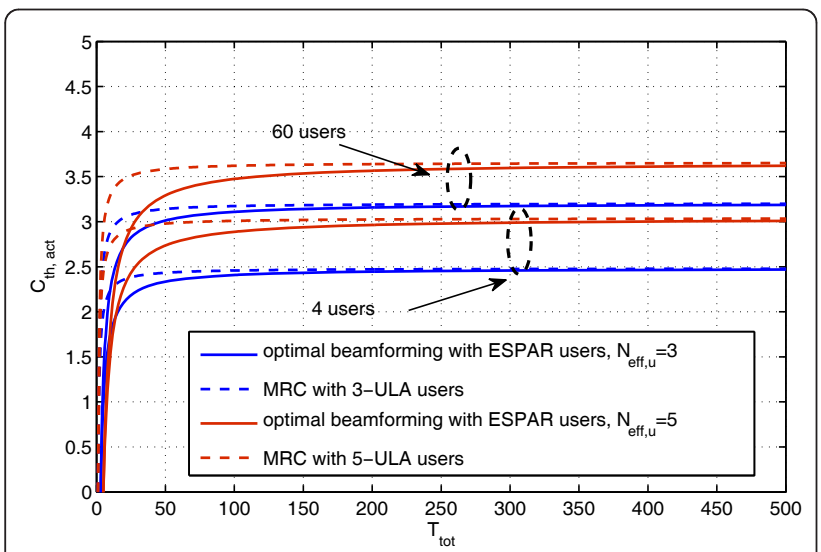

Figure 3 Training effect. Effect of training on the system performance. the basis pattern. Similarly, we define vector $\mathbf{p}_{u, R}$ for the receive radiation patterns. The LoS component has a phase shift $\varphi_{u}$ caused by propagation over the path connecting the $j$ th angle of departure and $i$ th angle of arrival. The amplitude of this component is naturally affected by the complex gains of the transmit and receive radiation patterns towards the same directions. In our approach, $\varphi_{u}$ is considered as a uniformly distributed random variable in the range of $[0,2 \pi)$.

Figure 4 shows the average throughput in opportunistic beamforming scenarios over Rician channels with factor $K=10$, in the case of using ESPAR antennas at the mobile receiver compared to the case of having conventional mobile terminals with a single antenna element. We identify two different cases, for $N_{\text {eff, } u}=N_{\text {eff }, T}$ $=3$ and 5 . As expected, the performance is enhanced when we use random directional patterns both at the BS and at the user terminals. Although we show only the case of $K=10$, it is evident from equation 13 that the artificial fading effects caused by random pattern switching will become more significant for higher Rician $K$ factors. This result is in agreement to the findings in the seminal paper of Tse [2] where the concept of opportunistic beamforming was introduced. In Figure 5, we show the effects of the Rician factor on the average throughput for the case of 32 users, normalized to the case where BS and users have a single antenna element and no beamforming capabilities.

\section{SINR optimization in MIMO broadcast channels}

In this section, we consider the case where the BS is capable of granting access to $U$ users simultaneously, by means of MU-MIMO broadcast channel. In this case, we are interested in the maximization of the signal to

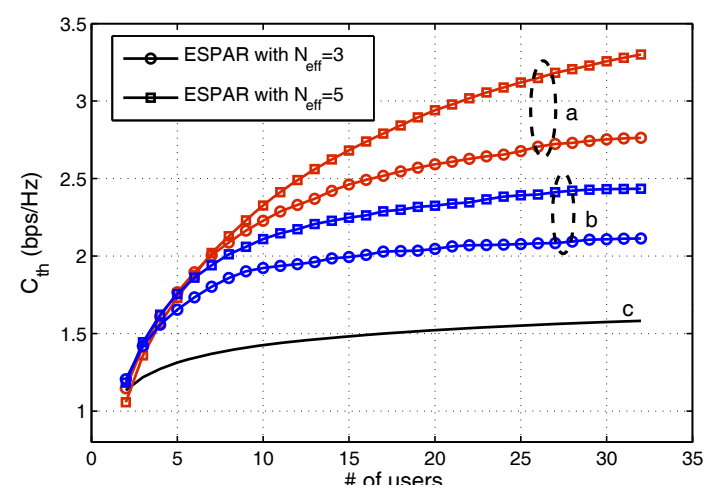

Figure 4 Average throughput with directive patterns. Average throughput when (a) directional random beams are used both at the BS and the user terminals, (b) directional random beams are used at the BS only, and (c) both the BS and the users are equipped with omni-directional antennas. 


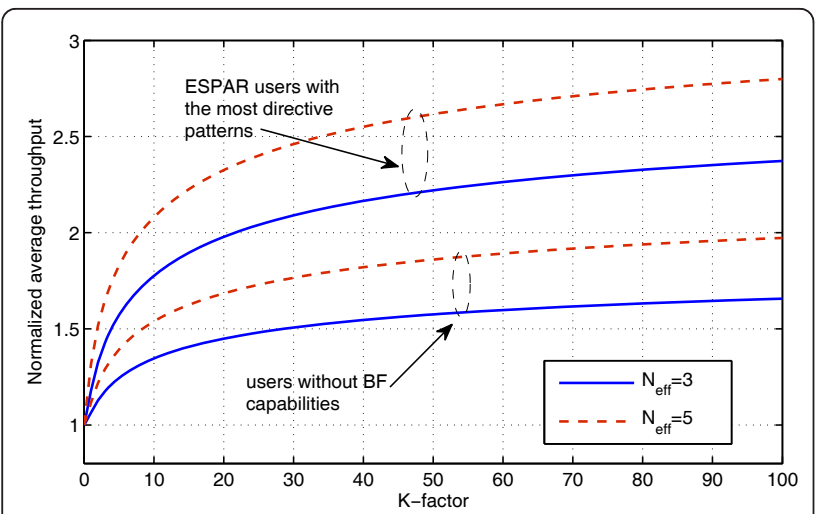

Figure 5 Normalized average throughput in Rician channels Performance of using ESPAR antennas at the users, normalized over the case where omni-directional antennas are used, with respect to the channel Rician factor. Thirty-two users are considered.

interference plus noise ratio at the user terminals, as considered also in $[29,30]$. The former publication is a generalization of the opportunistic beamforming technique, where a set of orthogonal radiation patterns is considered at the BS, each being assigned to a user with the maximum SINR. The orthogonal patterns are randomly assigned in each time slot, using an orthonormal precoding matrix, whose columns can be regarded as weighting vectors corresponding to orthogonal radiation patterns in the beam-space domain. The latter publication expands this concept by designing the orthogonal patterns according to the previous knowledge of the channels, rather than randomly. In this section, we propose an interference cancelation technique based on the use of parasitic arrays on the user terminals, exploiting the $N_{\text {eff, } u}$ DoFs of such antennas.

\subsection{An interference cancellation technique on user terminals}

We consider a broadcast channel of a multiuser environment where the BS and the mobile terminals are equipped with parasitic antennas of $N_{\text {eff, } T}$ and $N_{\text {eff, } u}$ DoFs, respectively, where in the general case, $N_{\text {eff, } T} \neq$ $N_{\text {eff, } u}$. The BS transmits simultaneously to $U \leq N_{\text {eff } T \text {. }}$. users on each time slot, and each user is assigned to a different basis transmit radiation pattern. Therefore, the BS functions as a MIMO transmitter with parasitic arrays as described for example in [9]. The users produce a linear combination of the $N_{\text {eff, } u}$ orthogonal patterns of their antenna in order to create the optimal receive pattern. This cancels out the interference caused by the $(U-1)$ simultaneous transmissions of the BS to the rest of the users, and at the same time maximizes the desired signal power. The ability of fulfilling these requirements depends on the number of DoFs $N_{\text {eff }, T}$ and $N_{\text {eff, } u}$, available on the BS and the user, respectively, as well as on the number of simultaneous users, $U \leq N_{\text {eff, } T \text {. }}$.

In the following, we assume that during the training period, each user $u$ acquires full knowledge of the $\mathbf{H}^{(u)}$ channel, with dimensions $\left(N_{e f f i} \times U\right)$. The elements $h_{i, j}^{(u)}$ of the channel matrix are the complex channel gains between the $j$ th transmit basis pattern and the $i$ th receive basis pattern. With this channel information each user may identify the transmit basis pattern that maximizes the SINR. Assume that the user has identified the $n$th transmit radiation pattern as such. The system model for this pattern will be,

$$
\gamma^{(u)}=\mathbf{w}_{u}^{H} \mathbf{H}^{(u)} \mathbf{s}+n^{(u)}=\underbrace{\mathbf{w}_{u}^{H} \mathbf{h}^{(u, n)} s^{(u)}}_{\text {use full signal }}+\underbrace{\sum_{i=1, i \neq u}^{U} \mathbf{w}_{u}^{H} \mathbf{h}^{(u, i)} \mathbf{s}^{(i)}+n^{(u)}}_{\text {interference }}
$$

where $\mathbf{H}^{(u)}=\left[\mathbf{h}^{(u, 1)} \mathbf{h}^{(u, 2)} \ldots \mathbf{h}^{(u, U)}\right]$, and the $\mathbf{h}^{(u, n)}$ vector expresses the complex gain between the $n$th transmit basis pattern and the set of receive basis patterns. Similarly, vectors $\mathbf{h}^{(u, i)}, i \neq u$ express interference. Vector $\mathbf{s}$ includes the transmission vectors to all users, and $\mathbf{w}_{u}$ is the weighting vector to produce the receive pattern in each user. The effective channel of user $u$ is $h^{u}=\mathbf{w}_{u}^{H} \mathbf{h}^{(u, n)}$. The SINR at the user terminal is given by:

$$
\begin{aligned}
\zeta_{u, n} & =\frac{\left|\mathbf{w}_{u}^{H} \mathbf{h}^{(u, n)}\right|^{2}}{\frac{1}{\gamma}+\sum_{i=1, i \neq n}^{U}\left|\mathbf{w}_{u}^{H} \mathbf{h}^{(u, n)}\right|^{2}}=\frac{\left(\mathbf{w}_{u}^{H} \mathbf{h}^{(u, n)}\right)\left(\mathbf{w}_{u}^{H} \mathbf{h}^{(u, n)}\right)^{H}}{\frac{1}{\gamma}+\sum_{i=1, k \neq n}^{U}\left(\mathbf{w}_{u}^{H} \mathbf{h}^{(u, i)}\right)\left(\mathbf{w}_{u}^{H} \mathbf{h}^{(u, i)}\right)^{H}} \\
& =\frac{\mathbf{w}_{u}^{H} \mathbf{D}_{u, n} \mathbf{w}_{u}}{\frac{1}{\gamma}+\mathbf{w}_{u}^{H} \mathbf{D}_{I} \mathbf{w}_{u}}=\frac{\mathbf{w}_{u}^{H} \mathbf{D}_{u, n} \mathbf{w}_{u}}{\frac{1}{\gamma}+\mathbf{w}_{u}^{H} \mathbf{H}_{I} \mathbf{H}_{I}^{H} \mathbf{w}_{u}}
\end{aligned}
$$

where

$$
\mathbf{D}_{u, n}=\mathbf{h}^{(u, n)}\left(\mathbf{h}^{(u, n)}\right)^{H}
$$

and

$\mathbf{D}_{I}=\sum_{i=1, i \neq n}^{U} \mathbf{h}^{(u, i)}\left(\mathbf{h}^{(u, i)}\right)^{H}=\sum_{i=1, i \neq n}^{U} \mathbf{D}_{u, i}=\mathbf{H}_{I} \mathbf{H}_{I}^{H} \quad$ have dimensions $\left(N_{\text {eff, } u} \times N_{\text {eff, }}\right)$, with rank one and $U-1$ respectively. The scalar term $\gamma$ corresponds to the average SNR at each user. $\mathbf{H}_{l}$ is formed by the $\mathbf{h}^{(u, i)}, i \neq u$ column vectors of the interference channels of user $u$. Note that $\operatorname{rank}\left(\mathbf{D}_{I}\right)=U$ - 1, i.e., is equal to the number of interfering signals.

The vector that will cancel interference is the one that belongs to the null space of matrix $\mathbf{H}_{l}$, since $\mathbf{w}_{u}^{H} \mathbf{h}^{(u, i)}=0, \forall i \neq n$. The orthonormal vectors of the null space can be derived either by applying the spectral theorem on the correlation matrix $\mathbf{D}_{I}$ in order to keep the vectors corresponding to zero eigenvalues, or by directly applying an eigenvalue decomposition to $\mathbf{H}_{I}$, 
keeping the vectors of the left orthonormal matrix that correspond to zero eigenvalues. With the former approach we will have $\mathbf{D}_{I}=\mathbf{U}_{I} \boldsymbol{\Lambda}_{I} \mathbf{U}_{I}^{H}$, where $\boldsymbol{\Lambda}$ is a $\left(N_{\text {eff, }} \times N_{\text {eff, } u}\right)$ diagonal matrix of the following form:

$$
\mathbf{\Lambda}_{I}=\operatorname{diag}\left(\tilde{\mathbf{\Lambda}}_{I} \mathbf{0}\right)
$$

where $\tilde{\mathbf{\Lambda}}$ is a $(U-1 \times U-1)$ matrix, while the null matrix $\mathbf{0}$ is a $\left(N_{\text {eff, } u}-U+1 \times N_{\text {eff, } u}-U+1\right)$ matrix. The vectors of the required null space are the right eigenvectors of $\mathbf{U}_{l}$ which correspond to the zero eigenvalues.

User $u$ can cancel out interfering signals when there exists at least one zero eigenvalue, or equivalently when there exists at least one null row in matrix $\boldsymbol{\Lambda}_{I}$. This is the case when $N_{\text {eff, } u} \geq U$, meaning that the user may cancel out interference whenever the DoFs of the receiving antenna are greater than or equal to the total number of users. We identify the following two cases:

- When $N_{\text {eff, } u}=U$, the null space has a single eigenvector (the first column of $\mathbf{U}_{\mathbf{1}}$ from the right), which can be used for interference cancelation.

- When $N_{\text {eff, } u}>U$ the null space has $N_{\text {eff, } u}-U+1$ eigenvectors.

In the latter case, any linear combination of the eigenvectors will also belong to the null space, therefore being able to cancel interference. We choose that linear combination, which will maximize the desired signal power, given by:

$$
\mathbf{w}_{u}=\sum_{i=1}^{N_{\text {eff }, u}-U+1} c_{u, i} \mathbf{u}_{I, i}
$$

where $\mathbf{u}_{I, i}$ are the $\left(N_{e f f, u}-U+1\right)$ eigenvectors defining the null space. The nominator of equation (15) will therefore become, due to (17):

$$
\begin{aligned}
\mathbf{w}_{u}^{H} \mathbf{D}_{u, n} \mathbf{w}_{u} & =\left(\sum_{i=1}^{N_{e f f, u}-U+1} c_{u, i} \mathbf{u}_{l, 1}\right)^{H} \mathbf{D}_{u, n} \sum_{i=1}^{N_{e f f, u}-U+1} c_{u, j} \mathbf{u}_{l, 1} \\
& =\left(\sum_{i=1}^{N_{e f f, u}-U+1} c_{u, i}^{*} \mathbf{u}_{l, 1}^{H}\right) \mathbf{h}^{(u, n)}\left(\mathbf{h}^{(u, n)}\right)^{H}\left(\sum_{i=1}^{N_{e f f, u}-U+1} c_{u, i} \mathbf{u}_{l, 1}\right) \\
& =\left(\sum_{i=1}^{N_{e f f, u}-U+1} c_{u, i}^{*} \mathbf{u}_{l, 1}^{H} \mathbf{h}^{(u, n)}\right)\left(\sum_{i=1}^{N_{e f f, u}-U+1} c_{u, i}\left(\mathbf{h}^{(u, n)}\right)^{H} \mathbf{u}_{l, 1}\right) \\
& =\left|\sum_{i=1}^{N_{e f f, u}-U+1} c_{u, i}\left(\mathbf{h}^{(u, n)}\right)^{H} \mathbf{u}_{l, 1}\right|^{2}=\left|\mathbf{c}^{H} \mathbf{r}\right|^{2}
\end{aligned}
$$

where

$$
\mathbf{c}=\left[c_{u, 1}^{*}, c_{u, 2}^{*}, \ldots\right]^{T}
$$

and

$\mathbf{r}=\left[\left(\mathbf{h}^{(u, n)}\right)^{H} \mathbf{u}_{I, 1},\left(\mathbf{h}^{(u, n)}\right)^{H} \mathbf{u}_{I, 2}, \ldots\right]^{T}$. It is therefore evident that the power of the desired signal is maximized when $\mathbf{c}=\mathbf{r} / k$, where $k=\|\mathbf{r}\|_{F}$. The use of the Frobenius norm to normalize $\mathbf{r}$ is required in order to ensure that $\|\mathbf{c}\|_{F}^{2}=1$.

According to (18), the optimal linear combination comes from the projection of the desired user channel on the null space vectors. From (17) and (18) it turns out that the optimal vector for canceling out interference while maximizing the desired signal strength is the following:

$$
\mathbf{w}_{u}=\frac{1}{k} \sum_{i=1}^{N_{\text {eff }, u}-U+1}\left[\left(\mathbf{h}^{(u, n)}\right)^{T} \mathbf{u}_{I, i}^{*}\right] \mathbf{u}_{I, i}, \quad U \leq N_{\text {eff }, T}
$$

We note that when $N_{\text {eff, } u}=U \leq N_{\text {eff, } T}$, (19) shows that the user can cancel out interference, but it is not able to maximize the desired signal strength, since the null space has a single eigenvector. Furthermore, if $N_{\text {eff, } u}<U$, then (19) does not hold, meaning that not all interfering signals can be canceled out. However, the user can still null out $\left(N_{\text {eff, } u}-1\right)$ stronger interfering signals, maximizing the SINR value for the available antenna capabilities.

\subsection{Evaluation of the proposed Scheme}

In the case of the MU-MIMO broadcast channel system under consideration, the average throughput of the system is given by [29]:

$$
C_{t h} \approx E\left\{\sum_{n=1}^{U} \log _{2}\left(1+\max _{u} \zeta_{u, n}\right)\right\}, \quad U \leq N_{\text {eff }, T}
$$

The equation above gives approximate results, since the probability that a user will have the optimal channel for more than one transmit radiation pattern, is not taken into consideration. However, we do include the cases for which $N_{\text {eff }, u}<U$, where the user will be able to null out only the $\left(N_{\text {eff, } u}-1\right)$ most significant interferences. In the results that follow, we consider parasitic arrays with $N_{\text {eff, } u}=3$ or 5 DoFs, while at the BS $N_{\text {eff, } T}=U$.

In Figure 6, we present the average throughput of the system, comparing the cases where the users are either equipped with parasitic arrays of $N_{\text {eff, } u}=3$ or with a conventional receiver having a single antenna element, as a function of the total number of users. It is evident that the proposed scheme performs significantly better than the current state of the art. It is noted that in the case where $U=2$, each user's null space consists of two orthonormal vectors, and it is therefore possible that each user may acquire the optimal radiation pattern that nulls out interference, while at the same time it maximizes the desired signal strength. The effect of this multi variable optimization is evident when comparing the cases where the users perform the full algorithm with the cases where only interference cancelation ("IC only" cases) is used. In the latter case, we remind the 


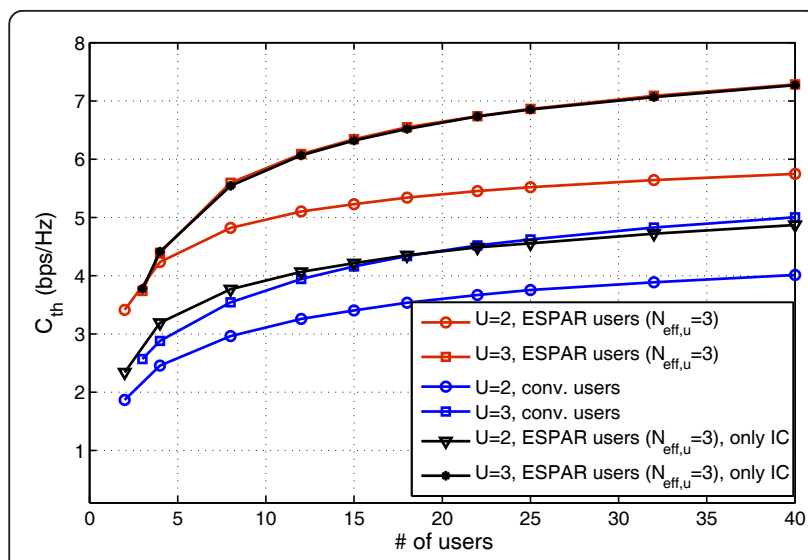

Figure 6 Average throughput with ESPAR with three DoF. System performance of the proposed scheme, compared to the case of users having conventional single-element antennas, $N_{\text {eff, } u}=3$.

reader that $\mathbf{w}_{u}$ is just one of the orthonormal vectors $\mathbf{u}_{I, i}$ of the null space.

As opposed to the case of $U=2$, where users may simultaneously cancel interference and maximize the desired signal's SNR, when $U=3$ the null space has a single eigenvector, which is used for interference cancelation, without any further optimization capabilities. This explains why the average throughput for $U=3$ and for ( $U=3$ "IC only" case) is the same. In Figure 7, we show the cumulative distribution function of the channel power. For $U=2$ the power of the channel is significantly higher due to the optimization capabilities of the antenna with $N_{\text {eff, } u}=3$. Finally, Figure 8 shows similar results for $N_{\text {eff, } u}=N_{\text {eff, } T}=3$ while Figure 9 shows the corresponding cdfs.

\section{Conclusions}

Conventional MU systems use either opportunistic beamforming or MU-MIMO in the broad-cast channel

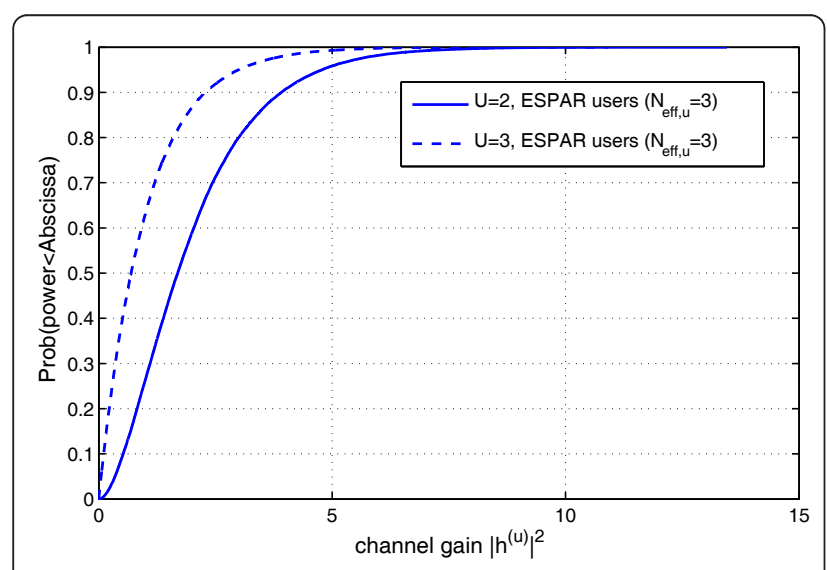

Figure 7 Channel power c.d.f. for three DoF. Cumulative distribution function of the channel power, $\mathrm{N}_{\text {eff, } u}=3$.

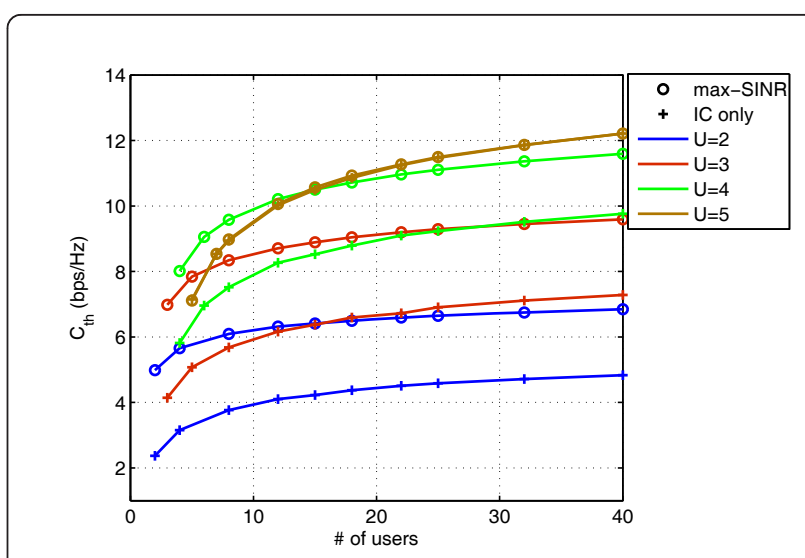

Figure 8 Average throughput with ESPAR with five DoF. System performance of the proposed scheme, compared to the case of users having conventional single-element antennas, $N_{\text {eff, } u}=5$.

and assume single antennas at the mobile station. We propose to take advantage of the developments in reconfigurable parasitic arrays in order to increase the performance of forward channels. The main idea is to integrate such antenna systems into mobile terminals, expand their beamspace domain into a basis of orthonormal radiation patterns and use this basis in the analysis for optimal beamforming and SINR optimization. The results show that in the case of opportunistic beamforming scenarios in Rayleigh channels only beamforming gains are achieved, as expected. In Rician channel environments the performance gains are significant and directly related to the $\mathrm{K}$-factor of the channel. In MUMIMO scenarios, the use of reconfigurable parasitic arrays at the receiver side significantly increases SINR and consequently the performance of the forward channel. Depending on the effective DoFs of the parasitic arrays and the total number of users, the receiver can cancel out all interfering signals, maximize channel

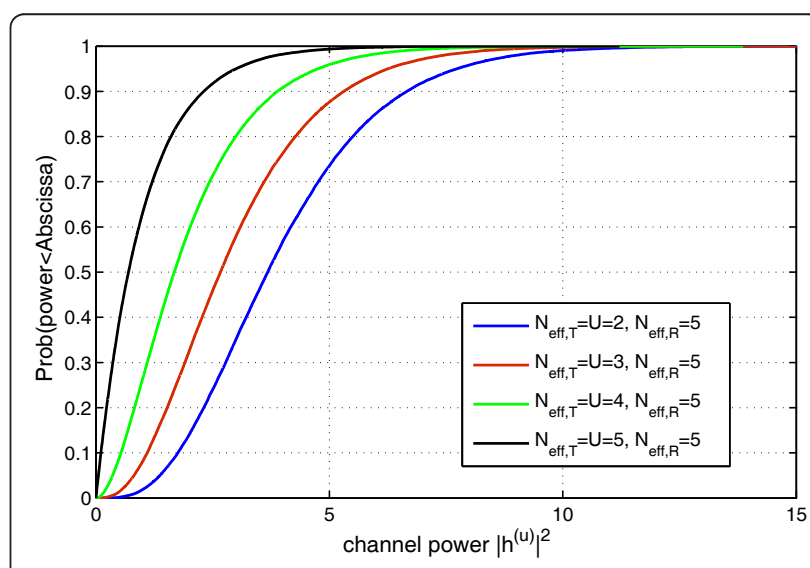

Figure 9 Channel power c.d.f. for five DoF. Cumulative distribution function of the channel power, $N_{\text {eff, } u}=5$. 
gains, or cancel out the most significant interferers. We therefore conclude that the use of parasitic arrays in multiuser scenarios shows considerable advantages, even in the case where the number of DoFs is limited due to implementation constraints of mobile terminals.

\section{Acknowledgements}

This work was partially funded by the European Union and national resources under the National Strategic Reference Framework (NSRF) and the THALES research project: INTENTION".

\section{Author details}

'Department of Digital Systems, University of Piraeus, 80 Karaoli \& Dimitriou St., 18534, Piraeus, Greece ${ }^{2}$ Athens Information Technology, $19.5 \mathrm{Km}$ Markopoulou Ave., 19002 Paiania, Attika, Greece ${ }^{3}$ Ecole Polytechnique Fédérale de Lausanne, ELB-037, EPFL-Station 11, CH-1015 Lausanne, Switzerland

\section{Competing interests}

The authors declare that they have no competing interests.

Received: 15 October 2011 Accepted: 3 February 2012

Published: 3 February 2012

\section{References}

1. R Knopp, PA Humblet, Information capacity and power control in single-cell multiuser communications, in IEEE International Conference on Communications, ICC, Seattle, 1, 331-335 (1995)

2. DN Tse, Optimal power allocation over parallel Gaussian broadcast channels, in IEEE International Symposium on Information Theory, IEEE, Ulm, Germany, 27 (29 Jun-4 July 1997)

3. P Viswanath, DNC Tse, Opportunistic beamforming using dumb antennas. IEEE Trans Inf Theory. 48(6), 1277-1294 (2002). doi:10.1109/TIT.2002.1003822

4. JD Boerman, JT Bernhard, Performance study of pattern reconfigurable antennas in MIMO communication systems. IEEE Trans Antennas Propag. 56, 231-236 (2008)

5. Z Li, Z Du, K Gong, Compact reconfigurable antenna array for adaptive MIMO systems. IEEE Antennas Wirel Propag Lett. 8, 1317-1320 (2009)

6. D Piazza, NJ Kirsch, A Forenza, RW Heath, KR Dandekar, Design and evaluation of a reconfigurable antenna array for MIMO systems. IEEE Trans Antennas Propag. 56(3), 869-881 (2008)

7. M Wennstrom, T Svantesson, An antenna solution for MIMO channels: the switched parasitic antenna, in 12th IEEE International Symposium on Personal, Indoor and Mobile Radio Communications, IEEE, San Diego, California, USA, 1, 159-163 (2001)

8. A Kalis, AG Kanatas, M Carras, AG Constantinides, On the performance of MIMO systems in the wavevector domain, in IST Mobile \& Wireless Comm Summit, Mykonos, Greece, (2006)

9. A Kalis, AG Kanatas, C Papadias, A novel approach to MIMO transmission using a single RF front end. IEEE J Sel Areas Commun. 26(6), 972-980 (2008)

10. ON Alrabadi, J Perruisseau-Carrier, A Kalis, MIMO transmission using a single RF source: theory and antenna design, IEEE Trans Antennas Propag, and IEEE Trans, Microwave Theory Tech., Joint Special Issue on MIMO Technology, accepted for publiccation, (2011)

11. O Alrabadi, C Papadias, A Kalis, R Prasad, A universal encoding scheme for MIMO transmission using a single active element for PSK modulation schemes. IEEE Trans Wirel Commun. 8(10), 5133-5142 (2009)

12. VI Barousis, AG Kanatas, A Kalis, Beamspace domain analysis of single RF front-end MIMO systems. IEEE Trans Veh Technol. 60(3), 1195-1199 (2011)

13. $V$ Barousis, A Kanatas, Aerial degrees of freedom of parasitic arrays for single RF front-end MIMO transceivers. Progress Electromag Res B. 35, 287-306 (2011)

14. V Elvira, J Via, Diversity techniques for RF-beamforming in MIMO-OFDM systems: design and performance evaluation, 17th European Signal Processing Conference (EU-SIPCO), EURASIP, Glasgow, Scotland, 1, 451-455 (2009)
15. F Gholam, J Via, I Santamaria, Beamforming design for simplified analog antenna combining architectures. IEEE Trans Veh Technol. 60(5), 2373-2378 (2011)

16. I Santamaria, V Elvira, J Via, D Ramirez, J Pérez, J Ibáñez Eickhoff, F Ellinger, Optimal MIMO transmission schemes with adaptive antenna combining in the RF path, in 16th European Signal Processing Conference (EUSIPCO), vol. 1. EURASIP, Lausanne, Switzerland, (2008)

17. J Via, V Elvira, I Santamaria, R Eickhoff, Analog antenna combining for maximum capacity under OFDM transmissions, in IEEE International Conference on Communications, ICC'09, IEEE, Dresden, Germany), 1-5 (2009)

18. J Via, V Elvira, J Ibáñez, I Santamaria, Optimal precoding for a novel REMIMO scheme in transmit correlated Rayleigh channels, in IEEE 10th Workshop on Signal Processing Advances in Wireless Communications, SPAWC'09, IEEE, Perugia, Italy, 334-338 (2009)

19. O Gónzalez Gutiérrez, J Ibàñez, L Vielva, R Eickhoff, Experimental evaluation of an RF-MIMO transceiver for 802.11a WLAN, in Future Network and Mobile Summit, Florence, Italy, 1-8 (2010)

20. NC Karmakar, in Handbook of Smart Antennas for RFID Systems, vol. chap. 20. (Wiley, 2010)

21. A Rajagopalan, G Gupta, AS Konanur, B Hughes, G Lazzi, Increasing channel capacity of an ultrawideband MIMO system using vector antennas. IEEE Trans Antennas Propag. 55, 2880-2887 (2007)

22. R Harrington, Reactively controlled directive arrays. IEEE Trans Antennas Propag. 26(3), 390-395 (1978). doi:10.1109/TAP.1978.1141852

23. C Su, A Hirata, T Ohira, NC Karmakar, Fast Beamforming of Electronically Steerable Parasitic Array Radiator Antennas: Theory and Experiment. IEEE Trans Antennas Propag. 52(7), 1819-1832 (2004). doi:10.1109/ TAP.2004.831314

24. VI Barousis, AG Kanatas, A Kalis, C Papadias, A stochastic beamforming algorithm for ESPAR antennas. IEEE Antenna Wirel Propag Lett. 7, 745-748 (2008)

25. FWJ Olver, DW Lozier, RF Boisvert, CW Clark, NIST Handbook of Mathematical Functions, (National institute of standards and technology (NIST) and Cambridge University Press, 2010)

26. G Strang, Linear Algebra and its Applications, 4th edn. (Brooks Cole, St. Paul, Minnesota, USA, 2005)

27. A Paulraj, R Nabar, D Gore, Introduction to Space-Time Wireless Communications, (Cambridge University Press, Cambridge, UK, 2003)

28. L Kahn, Ratio Squarer. Proc IRE Correspond. 42, 1074 (1954)

29. M Sharif, B Hassibi, On the capacity of MIMO broadcast channel with partial side information, in Thirty-Seventh Asilomar Conference on Signals, Systems and Computers, IEEE, Pacific Grove, CA, 2003, 2, 958-962

30. M Kountouris, D Gesbert, Memory-based opportunistic multi-user beamforming, in IEEE International Symposium on Information Theory, IEEE, Adelaide, Australia, pp. 1426-1430 (2005)

doi:10.1186/1687-1499-2012-30

Cite this article as: Barousis et al:: Reconfigurable parasitic antennas for compact mobile terminals in multiuser wireless systems. EURASIP Journal on Wireless Communications and Networking 2012 2012:30.

\section{Submit your manuscript to a SpringerOpen ${ }^{\odot}$ journal and benefit from:}

- Convenient online submission

- Rigorous peer review

- Immediate publication on acceptance

- Open access: articles freely available online

- High visibility within the field

- Retaining the copyright to your article

Submit your next manuscript at $\boldsymbol{s p r i n g e r o p e n . c o m ~}$ 